\title{
Correção cirúrgica de aneurismas da aorta torácica por técnica de exclusão
}

\author{
Bayard GONTIJO FILHO*, Juscelino T. BARBOSA*, João Alfredo Paula e SILVA*, Fernando A. FANTINI*,
} Marco Antônio SALUM*, Mário Osvaldo VRANDECIC*

RBCCV 44205-51

GONTIJO FILHO, B.; BARBOSA, J. T.; SILVA, J. A. P.; FANTINI, F. A.; SALUM, M. A.; VRANDECIC, M. O. - Correção cirúrgica de aneurismas da aorta torácica por técnica de exclusão. Rev. Bras. Cir. Cardiovasc., 3(1): 65-72, 1988.

RESUMO: São apresentados 14 pacientes portadores de aneurisma da aorta torácica ( 4 do arco aórtico e 10 da aorta descendente), com importantes complicaçōes pré-operatórias, que foram submetidos a correção cirúrgica através de técnica de exclusão da área aneurismática. Esta exclusão foi realizada através de um desvio extra-anatómico entre a aorta ascendente e a aorta abdominal, associado a ligadura da aorta, acima e abaixo do aneurisma. Em 5 pacientes, esta ligadura foi realizada com uso de suturas mecânicas (stapler). Houve $6(42,8 \%)$ óbitos no período pós-operatório, quase todos relacionados a grave condição clínica pré-operatória, em pacientes portadores de discussão aórtica aguda (tipo B). Dos 8 pacientes sobreviventes, 7 encontram-se em controle clínico por período de 6 meses a 4 anos, com boa evolução. Todos demonstraram redução progressiva do aneurisma, após a cirurgia, tendo, em alguns casos, ocorrido desaparecimento completo do mesmo.

DESCRITORES: aneurisma de aorta torácica, cirurgia.

\section{INTRODUÇÃO}

As inúmeras técnicas existentes para abordagem cirúrgica dos aneurismas da aorta torácica atestam a dificuldade do tratamento dessa doença. Essa dificuldade deve-se a vários fatores, tais como: localização anatômica da aorta torácica, particularmente do arco aórtico e da aorta descendente, que, especialmente na presença de aneurismas, tornam-se de difícil exposição; origem de ramos arteriais importantes na área lesada; graves alterações clínicas pré-operatórias, apresentadas por alguns pacientes; necessidade de emprego de circulação extracorpórea, em muitos deles, o que determina, em alguns casos, inconvenientes que podem contribuir para um mau resultado cirúrgico. Apesar destas dificuldades, os resultados da correção dos aneurismas do arco aórtico e da aorta descendente podem ser considerados bons, se analisarmos a gravidade dessa doença. Os maus resultados quase sempre referem-se a pacien- tes que se apresentam com graves complicaçōes, nos quais a mortalidade é significativamente aumentada. Nestes casos, a utilização de uma técnica cirúrgica baseada nas derivações extra-anatômicas, associada a exclusão do aneurisma, é uma boa alternativa. Nos últimos anos, empregamos esta técnica em 14 pacientes e a evolução desses casos é o motivo deste trabalho.

\section{MATERIAL E MÉTODO}

No período de janeiro de 1980 a dezembro de 1987, foram operados nos Serviços de cirurgia cardiovascular do Hospital Biocor e da Santa Casa de Misericórdia de Belo Horizonte, 60 pacientes portadores de aneurisma da aorta torácica. Diversas técnicas cirúrgicas foram empregadas nestes pacientes, observando-se, para isto, cada caso em particular. 
GONTIJO, FILHO, B.; BARBOSA, J. T.; SILVA, J. A. P.; FANTINI, F. A.; SALUM, M. A.; VRANDECIC, M. O. - Correção cirúrgica de aneurisma da aorta torácica por técnica de exclusão. Rev. Bras. Cir. Cardiovasc., 3(1): 65-72, 1988.

Desta série de 60 pacientes, 14 deles - sendo 4 portadores de aneurisma do arco aórtico e 10 portadores de aneurismas da aorta descendente - foram operados através de técnica de exclusão da área aneurismática. A idade dos pacientes variou de 32 a 75 anos, com a idade média de 55 anos.

Doze pacientes eram do sexo masculino, o que corresponde a $85 \%$ de todo o grupo. Os pacientes portadores de aneurisma do arco aórtico apresentavam complicaçōes pré-operatórias importantes, sendo que, em 1 deles, o aneurisma encontrava-se roto e, em outros 2, o processo aneurismático era extenso, acometendo, também, a aorta torácica descendente. (Figura 1). 0 outro paciente era portador de grave doença pulmonar, além de se apresentar em precário estado nutricional. Dez pacientes portadores de aneurisma da aorta torácica descendente foram divididos em 2 grupos: Grupo 1 aneurisma dissecante em fase aguda; Grupo 2 - aneurismas crônicos da aorta descendente. Os aneurismas operados na fase aguda (Grupo 1) foram em número de 7 , sendo que as indicaçōes para operação foram: rotura ( 2 casos), acometimento retrógrado do arco aórtico ( 2 casos), insuficiência renal aguda ( 1 caso), dor persistente num paciente portador de arco aórtico cervical e aorta descendente à direita ( 1 caso) e dor abdominal persistente (1 caso) (Figura 2).

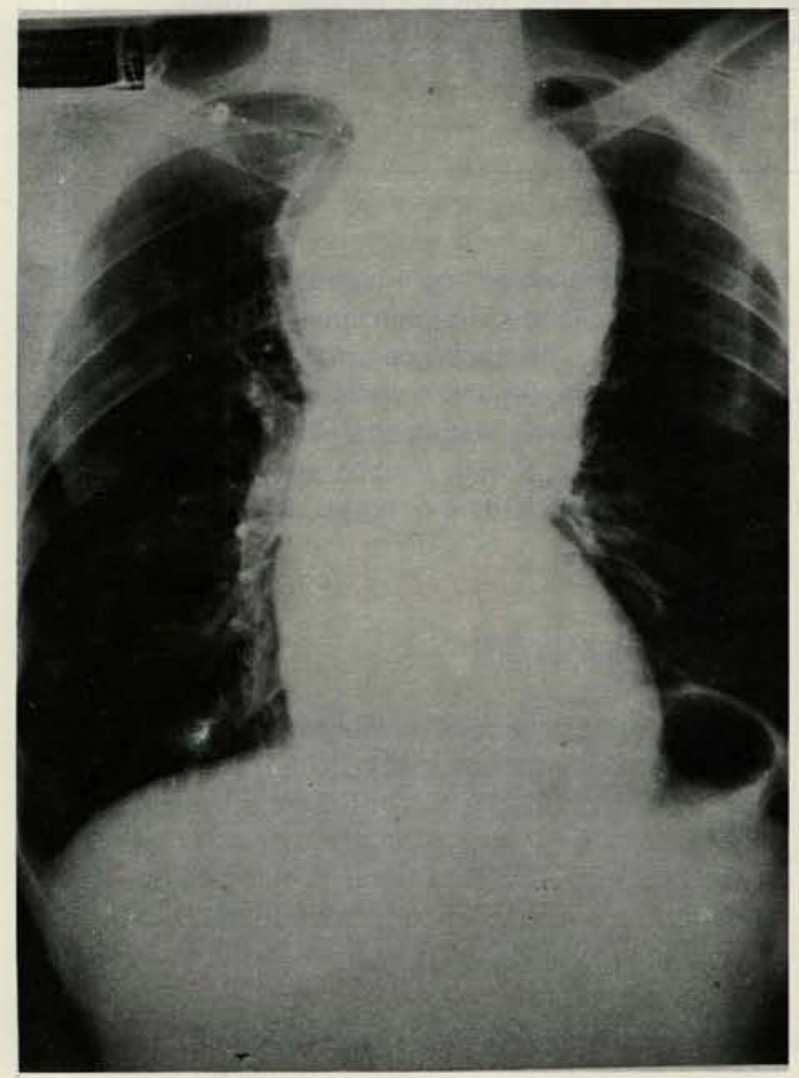

Fig. 1 - Aneurisma extenso de arco ab́rtico e aorta torácica descendente (Caso n: 14).

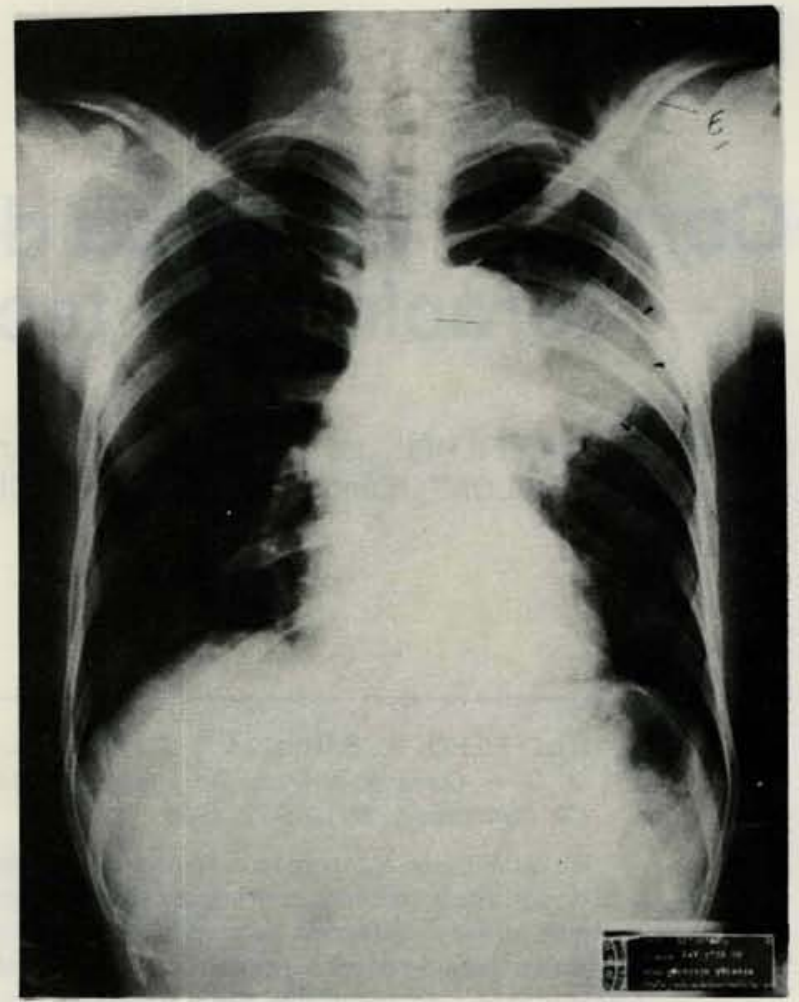

Fig. 2 - Aneurisma dissecante com hematoma e rotura intrapulmonar,

Os aneurismas do Grupo 2 foram operados por esta técnica por apresentarem graves problemas pulmonares associados.

Um paciente, que já havia sido submetido previamente a uma toracotomia exploradora, apresentava um enorme aneurisma acometendo praticamente toda cavidade torácica esquerda, com sinais de erosão para o brônquio. Os outros 2 pacientes apresentavam problemas pulmonares importantes, caracterizados por avançada doença pulmonar obstrutiva crônica (Figura 3 ).

\section{TÉCNICA CIRÚRGICA}

A técnica empregada, em todos os pacientes, foi basicamente a mesma, com algumas modificaçōes, devido a características próprias de cada caso. O acesso foi realizado por esternotomia prolongada ao abdome, através de uma laparotomia mediana. Após a escolha de uma prótese adequada, procedeu-se à anticoagulaçāo sistêmica (50 mg de heparina).

Inicialmente, realizou-se a anastomose proximal entre a prótese e a aorta descendente, através de exclusão parcial da aorta, mantendo-se controle farmacológico da pressāo arterial. Em seguida, a prótese foi passada para a cavidade abdominal, por um orifício no diagrama, e por tunelização retroperitonial, até o nivel da bifurcação 
GONTIJO, FILHO, B.; BARBOSA, J. T.; SILVA, J. A. P.; FANTINI, F. A.; SALUM, M. A.; VRANDECIC, M. O. - Correção cirúrgica de aneurisma da aorta torácica por técnica de exclusão. Rev. Bras. Cir. Cardiovasc., 3(1): 65-72, 1988.

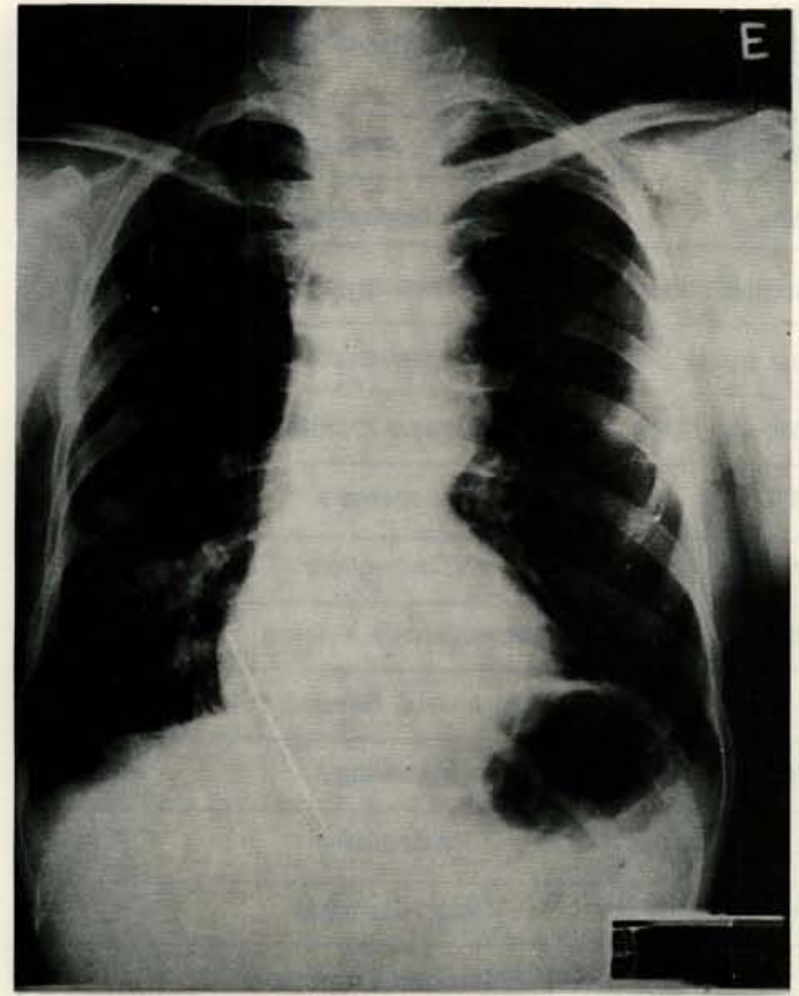

Fig. 3-Aneurisma de aorta descendente em paciente portador de grave acometimento pulmonar, principalmente do pulmăo direito.

da aorta, onde foi realizada a anastomose distal. Em 2 pacientes, utilizou-se a região supracelíaca da aorta abdominal, logo abaixo do diafragma, para realização da anastomose distal.

Uma vez estabelecido o circuito aorta ascendente-aorta abdominal, foram revascularizados os vasos supra-aórticos acometidos. Empregamos próteses retas para essa revascularização, geralmente anastomosadas ao implante previamente colocado na aorta ascendente. Finalmente, procedemos à oclusão da aorta acima e abaixo do aneurisma. Esta oclusāo foi realizada, nos primeiros pacientes, através de secção e sutura dos cotos aórticos; já nos últimos 5 pacientes, usamos sutura mecânica (stapler), para oclusão da luz arterial (Figura 4).

Em apenas 1 paciente, realizamos a oclusão somente da parte proximal, conforme técnica preconizada por CARPENTIER et alii ${ }^{3}$.

\section{RESULTADOS}

Desta série de 14 pacientes, $6(42,8 \%)$ vieram a falecer no período de pós-operatório imediato. As causas de óbito estão relacionadas no Quadro 1. Ressaltamos o óbito do único paciente (caso n: 4) submetido à técnica

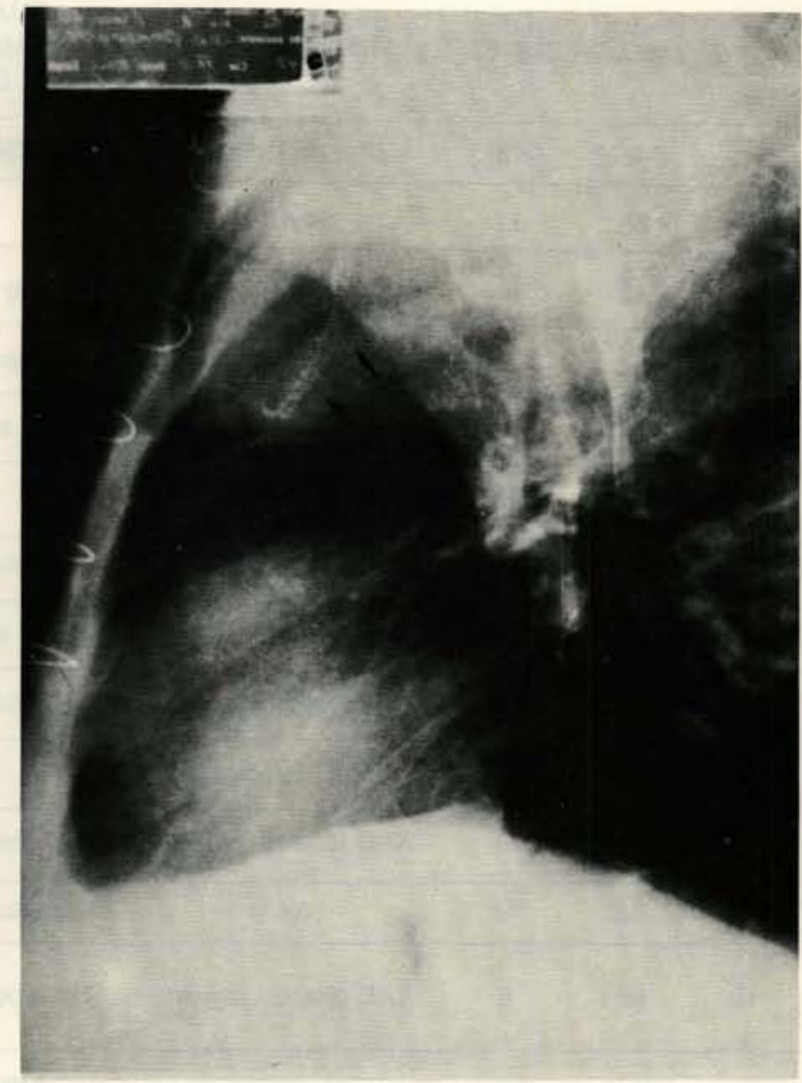

Fig. 4-Radiografia pós-operatória mostrando a presença de sutura mecânica (stapler) utilizada na oclusão aórtica proximal.

de Carpentier, que apresentou rotura aórtica no sègundo dia de pós-operatório.

Outra paciente, portadora de dissecção aórtica do tipo B acometendo toda a aorta abdominal (caso n: 11), evoluiu bem até a décima hora de pós-operatório, quando apresentou quadro de dor súbita, acompanhada de paraplegia e distensão abdominal, sendo realizado estudo angiográfico de urgência, que demonstrou integridade da técnica empregada, e quadro de redissecção retrógrada com hipofluxo dos vasos viscerais abdominais. A paciente veio a falecer antes de ser reoperada. Nos 8 pacientes sobreviventes, algumas complicaçōes foram encontradas no pós-operatório imediato, porém todas de aspecto temporário, com exceção de 1 paciente, que apresentou acidente vascular cerebral com seqüela posterior (hemiparesia esquerda) (caso $n: 6$ ). Dois pacientes apresentaram paresia do membro inferior esquerdo e 1 paciente teve paraparesia dos membros inferiores, todos com recuperação completa. Um paciente, portador de aneurisma de aorta descendente, apresentou quadro de febre prolongada no pós-operatório, que veio a ceder aproximadamente 35 dias após. O seguimento dos sobreviventes é de 6 meses a 4 anos, sendo que apenas 1 paciente não se apresenta em controle, atualmente. Seis pacientes foram submetidos a estudo angiográfico pós-operatório, que mostrou padrão vascular semelhan- 
GONTIJO, FILHO, B.; BARBOSA, J. T.; SILVA, J. A. P.; FANTINI, F. A.; SALUM, M. A.; VRANDECIC, M. O. - Correção cirúrgica de aneurisma da aorta torácica por técnica de exclusāo. Rev. Bras. Cir. Cardiovasc., 3(1): 65-72, 1988.

\begin{tabular}{|c|c|c|c|c|c|c|}
\hline \multicolumn{7}{|c|}{$\begin{array}{c}\text { DIAGNÓSTICO E EVOLUÇĀO DE } 14 \text { PACIENTES SUBMETIDOS A CORREÇÃO DE ANEURISMAS TORACICOS POR } \\
\text { TÉCNICA DE EXCLUSAOO }\end{array}$} \\
\hline №: & $I D A D E$ & SEXO & DIAGNÓSTICO & COMPL. PRÉ-OP. & COMPL. POS-OP. & EVOLUÇĀO \\
\hline 1 & 53 & M & AAA & Rotura & Insuf. respiratória & Óbito \\
\hline 2 & 42 & M & AAA & Extensão em Ao descendente & Obstr. subclávia (E) & Boa \\
\hline 3 & 75 & $\mathrm{~F}$ & AAD (dissec. agudo) & Insuf. renal aguda & IRA persistente & Óbito \\
\hline 4 & 58 & M & AAD (dissec. agudo) & Rotura em cavidade pleural & Rotura no $2:$ DPO & Óbito \\
\hline 5 & 67 & M & AAD (crônico) & DPOC & Monoparesia & Boa \\
\hline 6 & 32 & M & AAD (dissec. agudo) & Rotura em brônquio & AVC c/seqüela & Boa \\
\hline 7 & 45 & M & AAD (crônico) & Erosão em brônquio - Reop. & Monoparesia + febre & Boa \\
\hline 8 & 63 & M & AAD (dissec. agudo) & Dissecção retrógrada & IRA + insuf. respiratória & Óbito \\
\hline 9 & 64 & M & AAD (crônico) & DPOC & Nenhuma & Boa \\
\hline 10 & 51 & M & AAD (dissec. agudo) & Dissecção retrógrada & Paraparesia & Boa \\
\hline 11 & 35 & M & AAA & Extensāo em Ao descendente & Obstr. subclávia & Boa \\
\hline 12 & 68 & $\mathrm{~F}$ & AAD (dissec. agudo) & Dor abdominal persistente & $\begin{array}{l}\text { Redissecção + trombose } \\
\text { mesentérica }\end{array}$ & Óbito \\
\hline 13 & 58 & M & AAD (dissec. agudo) & $\begin{array}{l}\text { Arco Ao servical D } \\
\text { dor persistente }\end{array}$ & AVC & Óbito \\
\hline 14 & 60 & M & AAA & Mau estado geral - DPOC & Nenhuma & Boa \\
\hline
\end{tabular}

AAA - Aneurisma de aorta ascendente

$A A D$ - Aneurisma de aorta descendente

te, com bom funcionamento do circuito aorta ascendente-aorta abdominal e revascularização efetiva dos vasos cervicais, com exceção de 1 paciente, que apresentou oclusão da prótese utilizada para revascularização da artéria subclávia. Outro paciente (caso n: 11), portador de múltiplos aneurismas, que foi submetido, na primeira cirurgia, a ligadura das artérias subclávias, devido estarem acometidas por extenso processo aneurismático, foi reoperado 6 meses depois, procedendo-se a revascularização de ambos os membros superiores, através de implantes entre as carótidas e subclávias de ambos os lados (Figura 5).

O exame radiológico periódico do tórax, através de radiografia convencional, demonstrou uma reduçāo progressiva do tamanho do aneurisma, nos primeiros meses. Em alguns pacientes houve desaparecimento completo do aneurisma, o que, geralmente, ocorreu 1 ano após a cirurgia (Figuras 6 e 7).

Atualmente, apenas 2 pacientes encontram-se em classe funcional II, ambos em tratamento de hipertensão arterial, estando os demais assintomáticos.

\section{DISCUSSÃO}

O tratamento cirúrgico, a princípio, impóe-se a todo paciente portador de um aneurisma da aorta torácica, a não ser que exista uma contra-indicação formal à sua ressecção, já que a história natural desta doença é desfavorável. A cirurgia dos aneurismas foi, sem dúvida, um dos capítulos que mais desenvolvimento alcançou no tratamento das doenças cardiovasculares e, nos últimos 30 anos, muitas técnicas foram introduzidas e aperfeiçoadas, mas, ainda assim, os resultados não são os ideais, pois problemas, como sangramento, isquemia medular, insuficiência renal, acidente vascular cerebral e outros, ainda estão presentes em todas as grandes series cirúrgicas.

A conveniência de uma determinada técnica sobre a outra é ditada, basicamente, por 3 fatores: localizaçāo do aneurisma, condição clínica do paciente e experiência do grupo cirúrgico. Para correção de aneurismas do arco aórtico e alguns casos de aneurismas da aorta descendente, a hipotermia profunda e parada cardiocirculatória tem sido, ultimamente, técnica de eleição, em vários Serviços ${ }^{4}$. 
GONTIJO, FILHO, B.; BARBOSA, J. T.; SILVA, J. A. P.; FANTINI, F. A.; SALUM, M. A.; VRANDECIC, M. O. - Correção cirúrgica de aneurisma da aorta torácica por técnica de exclusão.

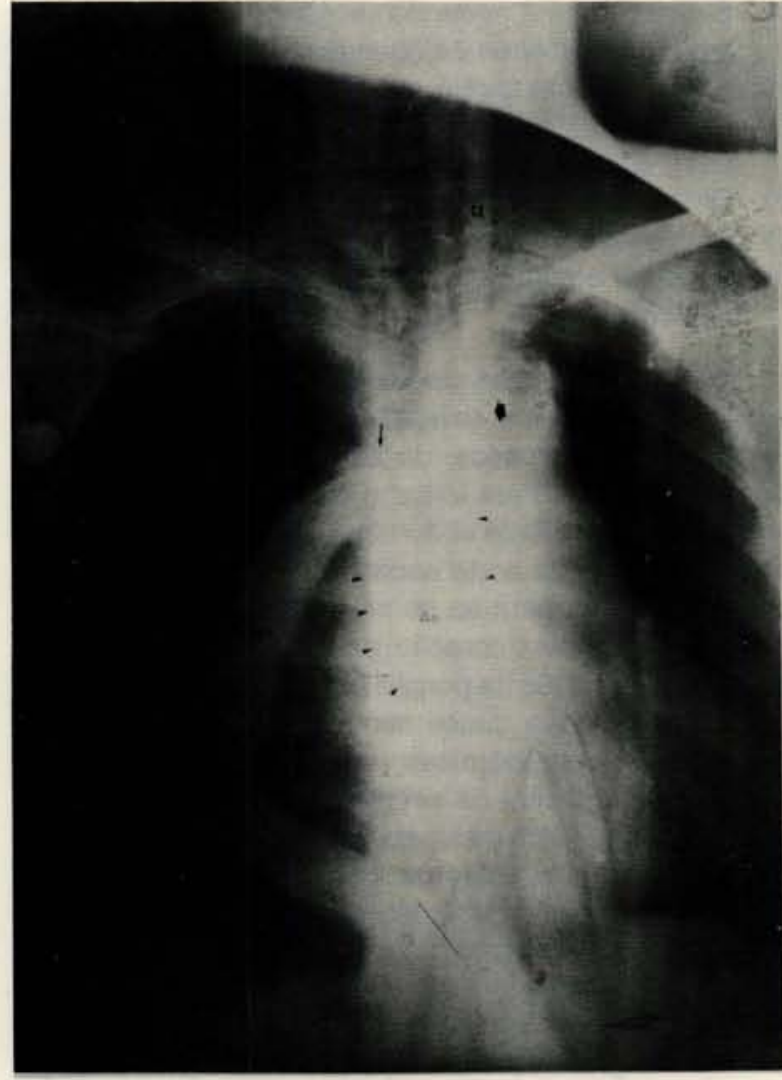

Fig. 5 - Angiografia pós-operatória mostrando a presença do by-pass aorta ascendente, aorta abdominal e interrupção do fluxo ao nivel do arco aórtico.

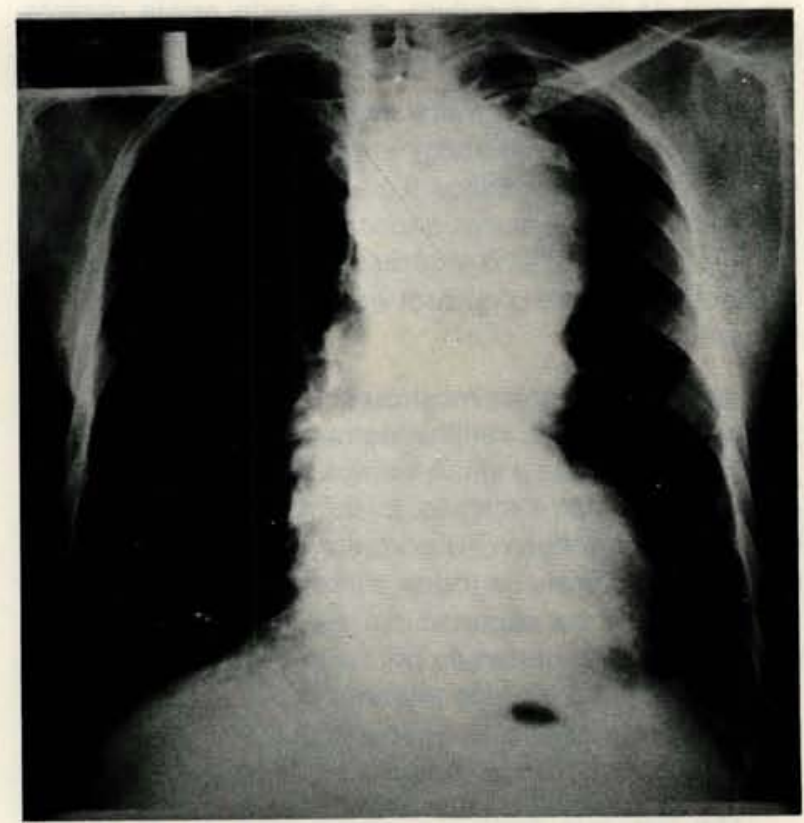

Fig. 6 - (B) - Controle radiológico no final do 2: ano pós-operatório demonstrando grande redução do saco aneurismático e recuperaçāo do parênquima pulmonar.

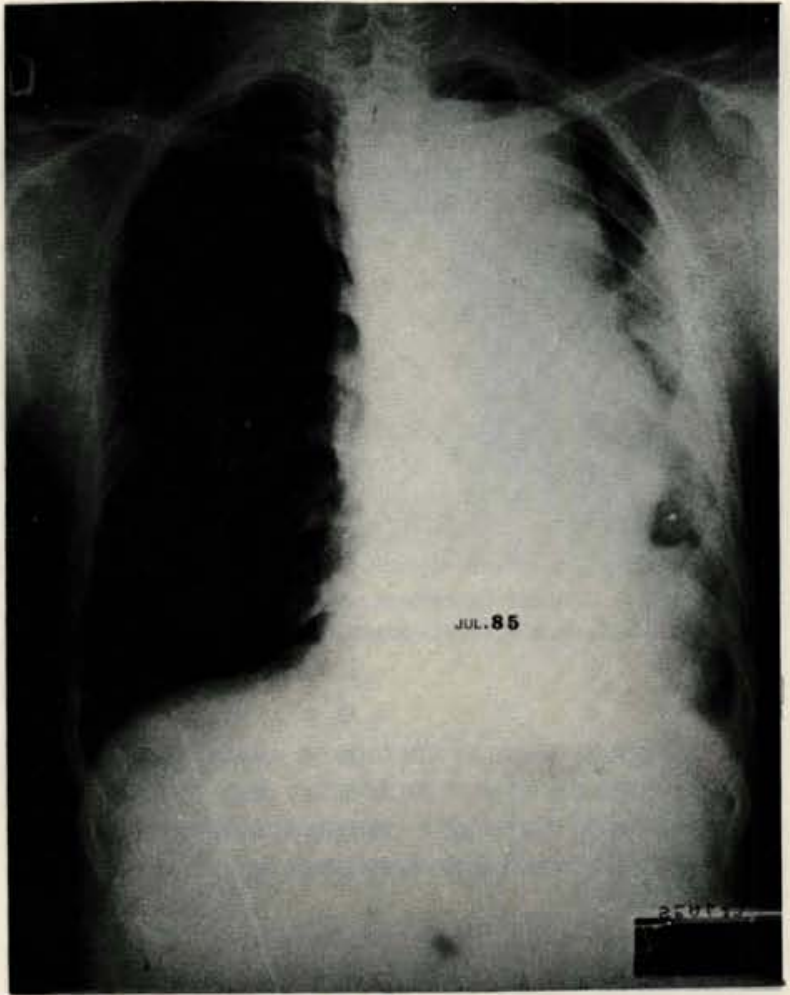

Fig. 6-(A) - Extenso aneurisma de aorta descendente com erosão brônquica e severo acometimento pulmonar (Caso n: 7).

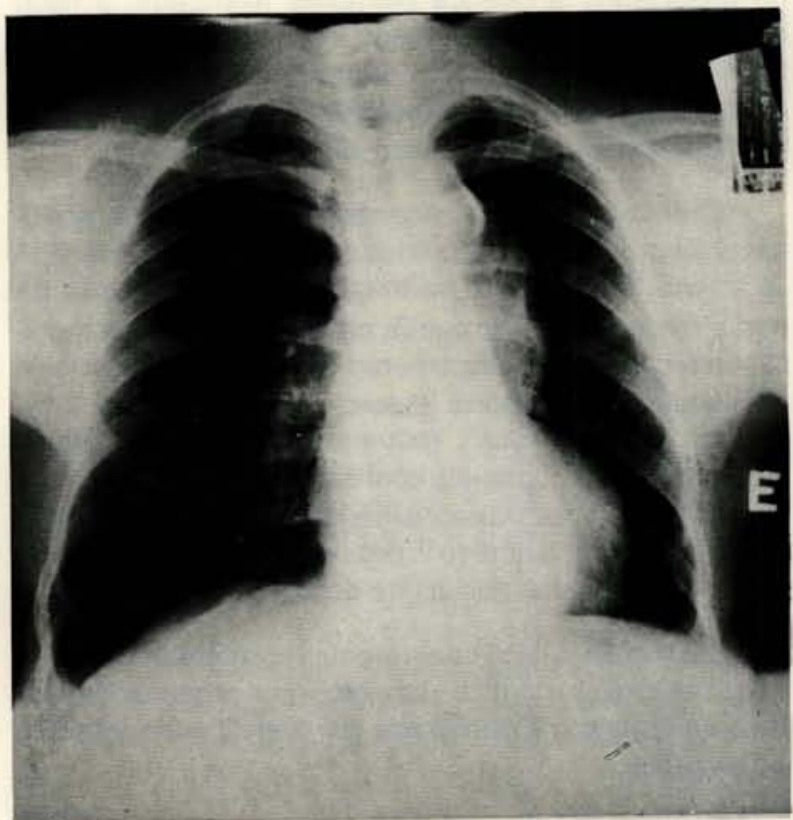

Fig. 7 - (A) - Aneurisma de aorta torácica descendente, tendo o estudo aortográfico demonstrado acometimento do arco aórtico (tronco bronquiocefálico e subclávia E). 
GONTIJO, FILHO, B.; BARBOSA, J. T.; SILVA, J. A. P.; FANTINI, F. A.; SALUM, M. A.; VRANDECIC, M. O. - Correção cirúrgica de aneurisma da aorta torácica por técnica de exclusāo. Rev. Bras. Cir. Cardiovasc., 3(1): 65-72, 1988

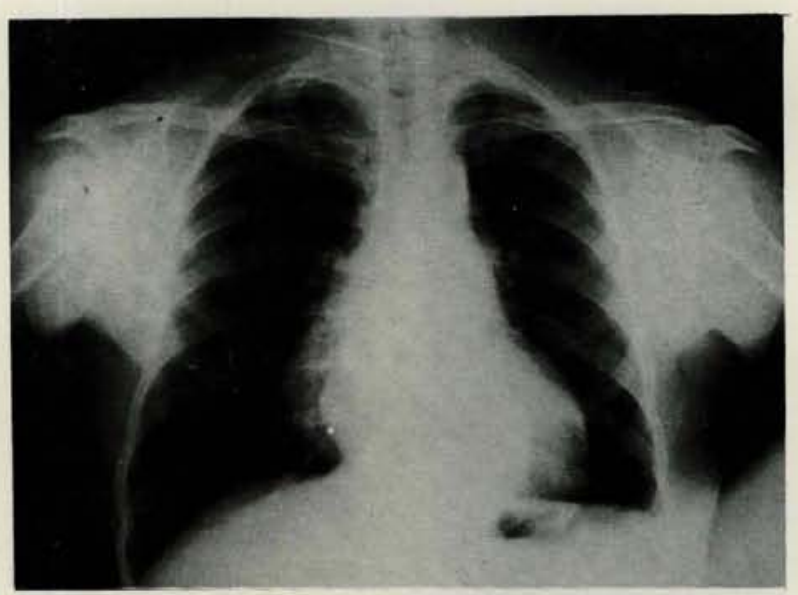

Fig. 7- (B) - Controle radiológico pós-operatório (1 ano) demonstrando desaparecimento quase total do aneurisma.

Não obstante suas vantagens, acreditamos que o método ainda apresenta problemas que, muitas vezes, se tornam de difícil controle, particularmente em Serviços menores, cujo movimento com esse tipo de lesão é restrito.

Quanto aos aneurismas da aorta torácica descendente, existem, basicamente, 3 técnicas que dividem a preferência dos grupos cirúrgicos: 1) ressecção com pinçamento simples da aorta, sem proteçāo visceral; 2) ressecção com emprego de circulação extracorpórea, ou shunt externo (Gott); 3 ) interposição de prótese intraluminal. Sem entrar no mérito de cada uma delas, podemos dizer que, em algumas situaçōes clínicas, tais como a dissecção aguda e aneurismas crônicos complicados ou associados a doença pulmonar avançada, o risco cirúrgico torna-se aumentado com qualquer técnica. Com o intuito de diminuir a mortalidade e a morbidade, nestas situaçōes, foram propostas técnicas baseadas em reconstrução aórtica extra-anatômica ${ }^{3}$.

O desvio da aorta ascendente para a aorta abdominal foi proposto, pela primeira vez, por SCHUMAKER Jr. et. alii ${ }^{12}$, em 1968, no tratamento da coartação da aorta. Posteriormente, outros autores utilizaram a técnica, com sucesso, no tratamento de outras doenças, tais como: estenose da aorta torácica descendente por arterites ou aterosclerose $e^{5}$, recoartaçāo da aorta ${ }^{14}$, coartação da aorta abdominal, coartação da aorta associada a outras doenças ${ }^{8}$, aneurisma da aorta descendente, aneurisma do arco aórtico ${ }^{13}$, próteses infectadas da aorta torácica ${ }^{9}$ e casos especiais de oclusão aorto-iliaca ${ }^{7}$.

O princípio básico desta técnica consiste em estabelecer um circuito aórtico paralelo, que proporciona um fluxo anterógrado e retrógrado ao nível da aorta torácica e abdominal.

Desta forma, o segmento aórtico afetado poderá ser excluído da circulação. As principais vantagens dạ técnica são:
1) É muito versátil, podendo ser aplicada no tratamento de diferentes tipos de doenças.

2) Evita o acesso direto à área lesada, possibilitando ao cirurgião trabalhar em áreas livres e com tecidos em melhores condiçōes de sutura.

3) Evita a manipulação do parênquima pulınonar.

4) Dispensa o uso de circulação extracorpórea.

5) Pode ser empregada com pouca ou nenhuma heparinizaçāo.

São duas as suas desvantagens principais: a presença da prótese na cavidade abdominal e a necessidade, em alguns casos, de se realizar maior número de anastomoses. Para evitar o contato da prótese com os órgãos da cavidade abdominal, alguns autores preconizam o desvio da aorta ascendente para a aorta torácica. Entretanto, para se conseguir tal intento é necessário que se retraia o coração, para realização da anastomose distal, através da porção posterior do saco pericárdico. Esta retração quase sempre é acompanhada de alterações hemodinâmicas importantes, impondo-se, muitas vezes, o uso de circulação extracorpórea para sua realização. Além disto, acreditamos que o posicionamento correto da prótes na cavidade abdominal evita os inconvenientes do seu contato direto com os órgãos ocos, que podem sofrer erosão em uma fase mais tardia. Quando a anastomose distal é feita ao nivel da bifurcaçāo da aorta, o implante é passado por um túnel retroperitoneal, emergindo ao nível da pequena curvatura do estômago e, através de orifício no diafragma, atinge a aorta descendente. Se a anastomose é feita na região supracelíaca da aorta, é importante isolar a área da sutura com os próprios tecidos adjacentes, ou, mesmo, com um pedículo omental. Desta forma, pensamos que a erosão será evitada, já que a mesma ocorre quase sempre ao nível da linha de sutura, ou quando existe contato direto da prótese com as vísceras ocas'.

A exclusão do segmento aórtico é feita com oclusāo da aorta acima e abaixo do aneurisma, isolando-o, assim, da circulação. Nos últimos 5 pacientes, utilizamos suturas mecânicas para oclusão da luz aórtica, conforme preconizado por alguns autores ${ }^{2,6}$, substituindo a técnica de secção e chuleio que foi empregada nos primeiros pacientes.

A sutura mecânica mostrou-se efetiva para esta finalidade e os 2 óbitos verificados nestes 5 pacientes não foram relacionados a ela. A técnica preconizada por Carpentier, de trombo-exclusão, foi utilizada em um dos nossos pacientes (caso n: 4) portador de dissecção aguda da aorta com sinais de rotura intrapleural. Este paciente veio a falecer no segundo dia de pós-operatório, por rotura, que foi comprovada por necrópsia. Apesar dessa complicação nāo ter sido relatada por Carpentier, julgamos prudente não empregar a técnica de trombo-exclusão em pacientes portadores de aneurismas rotos.

Um fato que deve ser considerado, ao se utilizar essa técnica, é a possibilidade de dissecção retrógrada ao se estabelecer o fluxo pelo circuito aorta ascenden- 
GONTIJO, FILHO, B.; BARBOSA, J. T.; SILVA, J. A. P.; FANTINI, F. A.; SALUM, M. A.; VRANDECIC, M. O. - Correção cirúrgica de aneurisma da aorta torácica por técnica de exclusão. Rev. Bras. Cir. Cardiovasc., 3(1): 65-72, 1988.

te-aorta abdominal. Acreditamos que este processo tenha sido causa de acidente vascular cerebral em 2 casos da nossa série, tendo ambos ocorrido em pacientes com dissecção aguda. Uma outra paciente (caso n: 12) apresentou quadro de redissecção retrógrada com oclusão de vasos abdominais no 1: dia de pós-operatório, o que foi confirmado angiograficamente. Nos casos em que houver dissecção aórtica até a sua bifurcação, sugerimos que a anastomose distal seja feita com ancoramento externo de feltro, ou que se utilize a cola biológica.

Mesmo assim, podemos verificar que dissecçōes agudas da aorta descendente ainda persistem com alto índice de mortalidade, sendo que 5 , dos 6 óbitos da nossa série, ocorreram em pacientes com essa grave doença.

A probabilidade de isquemia medular existe após a exclusão de segmentos da aorta torácica, da mesma forma que nas técnicas convencionais, em que não se emprega a revascularização de artérias intercostais. Nos nossos pacientes, a isquemia medular se traduziu apenas por episódios transitórios de paresia dos membros inferiores em 3 pacientes. Outras complicações citadas, na literatura, como secundárias a essa técnica, como sobrecarga ventricular esquerda ${ }^{10}$ e coagulação intravascular disseminada ${ }^{11}$, não foram observadas por nós.

Considerando a gravidade dos 14 pacientes analisados nesta série, concluímos que a técnica de exclusāo aórtica foi uma alternativa válida, principalmente nos pacientes com aneurismas crônicos complicados do arco aórtico e da aorta torácica descendente. Nos casos de dissecçōes agudas (tipo B), a mortalidade persistiu elevada, năo mostrando esse procedimento superioridade sobre as técnicas convencionais.

\section{RBCCV 44205-51}

GONTIJO FILHO, B.; BARBOSA, J. T.; SILVA, J. A. P.; FANTINI, F. A.; SALUM, M. A.; VRANDECIC,

M. O. - Surgical correction of aneurysms of the thoracic aorta using the aneurysmal exclusion technique. Rev. Bras. Cir. Cardiovasc., 3(1): 65-72, 1988.

ABSTRACT: The authors report their experience with 14 patients who underwent correction of aneurysms of the aortic arch and descending thoracic aorta, utilizing a technique based on aortic exclusion. The approach was accomplished with an ascending abdominal aortic by-pass, and the aneurysm was isolated with a ligature of the aorta above and below it. In 5 patients, this ligature was done with staplers. Six patients died in the immediate post-operative period, mainly from preoperative clinical condition related to acute aortic dissection of the descending thoracic aorta. Sevem patients have been followed from 6 months to 4 years and all of them showed a progressive reduction of the aneurysmal sac.

DESCRIPTORS: aneurysms, thoracic aorta, surgery.

\section{REFERÊNCIAS BIBLIOGRÁFICAS}

1 BERRY B. E. \& QUARD, R. P. - Left ventricular abdominal aortic conduit complicated by late gastric erosion. J. Thorac. Cardiovasc. Surg., 82(1): 147-149, 1981.

2 BlumenBeRG, R. M. \& Gelfand, M. L. - Application of intestinal staplers to aorto iliac surgery. $A m$. J. Surg., 144(2): 198-202, 1982.

3 CARPENTER, A.; DELACHE, A.; FABIANO, L.; CHAVAUD, S.; RELLAND, J.; NOTTIN, R.; VOUHÉ, P. L.; MASSOUD, H.; DUBOST, C. - New surgical approach to aortic dissection: flow reversal and thromboexclusion. J. Thorac. Cardiovasc., Surg., 81(5): 659-668, 1981.

4 CRAWFORD, E. S. \& SNYDER, D. M. - Treatment of aneurysms of the aortic arch: a progress report. J. Thorac. Cardiovasc. Surg., 85(2): 237-246, 1983.

5 DUNCAN, J. M. \& COOLEY, D. A. - Surgical considerations aortitis with special emphasis on Takayasu's arteritis. Texas Heart Inst. J., 10(3): 233-247, 1983.
6 ERGIN, M. A.; O'CONNOR, J. V.; BLANCHE, C.; BRIEPP, R. B. - Use of stapling instruments in surgery for aneurysms of the aorta. Ann. Thorac, Surg., 36(2): 161-166, 1983.

7 FRANTZ, S. L. \& KAPLITT, M. J. - An alternate operative approach for the highly disease of abdominal aorta. Bull. Texas Heart Inst. 3(1): 191-193, 1974.

8 GONTIJO FILHO, B; FANTINI, F. A.; SILVA, J. A. P.; BARBOSA, J. T.; SIMÓES, R.; FREIRE, R. J. A.; - Tratamento cirúrgico simultâneo de coartação de aorta e de insuficiência coronária: relato de caso. Arq. Bras. Cardiol., 41(6): 459-462, 1983.

9 HARGROVE, W. C. \& EDMUNDS Jr., L. H. - Managemerit of infected thoracic aortic prosthetic grafits. Ann. Thorac. Surg., 37(1): 72-77, 1984.

10 MITSUI, T.; MAETA, H.; FUKUDA, I.; IGIMA, H.; OKAMURA, K.; SAKAI, A.; SAKURAI, J.; HORI, M. - Left ventricular hypertrophy due to aortic bypass grafting with a long prosthesis. J. Cardiovasc. Surg., 27(2): 201-206, 1986. 
GONTIJO, FILHO, B.; BARBOSA, J. T.; SILVA, J. A. P.; FANTINI, F. A.; SALUM, M. A.; VRANDECIC, M. O. - Correção cirúrgica de aneurisma da aorta torácica por técnica de exclusão. Rev. Bras. Cir. Cardiovasc., 3(1): 65-72, 1988.

11 MORISHILA, Y.; TABETA, F.; SAIGENJI, H.; TAIRA, A. - Disseminated intravascular coagulopathy associated with with thrombo exclusion for dissecting aortic aneurysm. J. Cardiovasc. Surg., 27(4): 731-733, 1986.

SCHUMAKER Jr., H. B.; NAHRWOLD, D. L.; KING, H.; WALDHAUSEN, J. A. - Coarctation of the aorta. Curr. Probl. Surg., 16: 1-64, 1968.
13 URSHEL, H. C.; RAZZUK, M. A.; LESHNOWER, A. C. - Bypass grafting and aneurysmorraphy for aortic arch aneurysms. Ann. Thorac. Surg., 35(6): 579-583, 1983.

14 WUKASCH, D. C.; COOLEY, D. A.; SANDIFORD, F. M; NAPPI, G.; REUL Jr., G. J. - Ascending aorta-abdominal aorta bypass: indications, technique and report of 12 patients. Ann. Thorac. Surg., 23(5): 442-448, 1977. 